\title{
Klotho inhibits the capacity of cell migration and invasion in cervical cancer
}

\author{
BOOGI CHANG ${ }^{1 *}$, JINSUN KIM $^{1 *}$, DONGJUN JEONG ${ }^{2 *}$, YUJUN JEONG $^{2}$, SEOB JEON $^{3}$, SAM-IL JUNG ${ }^{1}$, \\ YOUNG YANG ${ }^{1}$, KEUN IL KIM ${ }^{1}$, JONG-SEOK LIM ${ }^{1}$, CHANGJIN KIM ${ }^{2}$ and MYEONG-SOK LEE ${ }^{1}$ \\ ${ }^{1}$ Research Center for Women's Diseases and Department of Biological Sciences, Sookmyung Women's University, \\ Seoul; ${ }^{2}$ Department of Pathology, College of Medicine, Soonchunhyang University; ${ }^{3}$ Department of \\ Obstetrics and Gynecology, Soonchunhyang University Hospital, Cheonan, Republic of Korea
}

Received March 27, 2012; Accepted May 22, 2012

DOI: $10.3892 /$ or.2012.1865

\begin{abstract}
Aberrant activation of the Wnt/ $\beta$-catenin signaling pathway is common in human cervical cancers. However, the mechanisms of Wnt activation in cervical cancer remain largely unknown. In the present study, we demonstrate that Klotho, a Wnt antagonist, is downregulated in invasive human cervical tumors and in a cell line we analyzed. Our data demonstrated that in vivo Klotho expression was not observed in invasive cervical carcinoma. In vitro restoration of Klotho expression in SiHa cells resulted in a decreased cell motility and invasiveness through upregulation of E-cadherin, downregulation of $\mathrm{N}$-cadherin and reduced expression of MMP7 and -9. Ectopic expression of Klotho also reduced the expression of the epithelial-to-mesenchymal transition (EMT) transcription factors Slug and Twist. Furthermore, Klotho causes a significant inhibition of the Wnt/ $\beta$-catenin pathway in cervical cancer cells, as supported by the expression of Wnt/ $\beta$-catenin transcriptional target genes such as c-Myc and cyclin D1. Consequently, our findings demonstrate for the first time that Klotho regulates tumor invasion through the EMT process and provide novel mechanistic insights into the role of Klotho in cervical cancer progression and contribute to treatment for metastatic cervical cancer patients.
\end{abstract}

Correspondence to: Dr Myeong-Sok Lee, Department of Biological Sciences, Sookmyung Women's University, 53-12 Chungpa-Dong 2-ka, Yongsan-Ku, Seoul 140-742, Republic of Korea

E-mail: mslee@sookmyung.ac.kr

*Contributed equally

Abbreviations: HPV, human papilloma virus; Fz/LRP, Frizzled/ low-density lipoprotein receptor-related protein; TCF/LEF, T-cell factor/lymphoid enhance factor; IGF-1, insulin-like growth factor-1; FGF23, fibroblast growth factor 23; EMT, epithelial-tomesenchymal transition; MMPs, matrix metalloproteinases; ABC, active form of $\beta$-catenin

Key words: Klotho, cervical cancer, Wnt/ $\beta$-catenin pathway, metastasis, epithelial-to-mesenchymal transition

\section{Introduction}

Cervical carcinoma is one of the most common cancers and the second-leading cause of cancer deaths in women worldwide (1). Substantial research has been performed to identify the causative agents for development of cervical cancer and now it is generally accepted that human papilloma virus (HPV) is the principal etiological agent of cervical cancer (2). Although the virus infecting these tumors can immortalize human cells, it does not result in transformation. Therefore, HPV infection is likely to be necessary, but insufficient for developing cervical cancers. It might mean there are factors epigenetic, genetic, cellular, and environmental that can influence carcinogenesis (3). Although the precise molecular mechanisms are still unclear, the most possible signaling pathways that is considered as the second hit in the multistep process of cervical carcinogenesis caused by HPV is the Wingless-type (Wnt)/ $\beta$ catenin pathway $(4,5)$.

The Wnt pathway is an important regulator in the control of several biological processes such as proliferation and differentiation in embryogenesis, regulation of the cell cycle, tissue homeostasis in adult tissue and tumor progression (6). Wnt ligands binding to its receptor complex comprised of Frizzled/ low-density lipoprotein receptor-related protein (Fz/LRP) trigger a canonical pathway. In this pathway, $\beta$-catenin was stabilized by inhibition of its phosphorylation and subsequent proteosomal degradation. Stabilized $\beta$-catenin translocates to the nucleus and forms a complex with T-cell factor/lymphoid enhancer factor (TCF/LEF) to activate target genes (7). In contrast, Wnt inhibition caused by its antagonists leads to decreased accumulation of cytosolic and nuclear $\beta$-catenin with consequent downregulation of Wnt reactive genes.

Aberrant activation of the Wnt/ $\beta$-catenin signaling pathway contributes to the progression of several major human cancers (6). Cytoplasmic and nuclear accumulation of $\beta$-catenin is the main hallmark of Wnt activation and is observed in most cervical cancer specimens (8). However, mutations of APC and $\beta$-catenin genes that are usually responsible for the deregulated Wnt/ $\beta$-catenin pathway in other tumors are rare in human cervical cancer $(8,9)$. It suggests that Wnt activation is the main regulator of $\beta$-catenin in cervical cancer. Supporting this hypothesis, promoter hypermethylation of characteristic Wnt 
antagonists Dickkopf-3, secreted Frizzled related protein-1, $-2,-4$ and Klotho has been identified in cervical carcinoma $(10,11)$.

Klotho was first identified as a potent suppressor of aging, so loss of Klotho can result in multiple aging-like phenotypes (12). Klotho is a 1012-amino acid single pass transmembrane protein, and its extracellular domain can be cleaved, shed into the serum, and it can act as a circulating hormone $(13,14)$. The intracellular domain is short and no known functional domains exist. Besides of its principle $\beta$-glucosidase activity $(12,15)$, Klotho is involved in multiple biological processes. It is now generally accepted that Klotho inhibits insulin and insulin-like growth factor (IGF-1) signaling and acts as a co-receptor for fibroblast growth factor 23 (FGF23) (16). Multiple lines of evidence proposed that the expression level of Klotho influences human breast, and lung cancers via intervention of IGF-1 and insulin pathway $(17,18)$. However, there are not sufficient studies on the role of Klotho in cervical cancer progression. In our previous study (19), we showed epigenetic silencing of Klotho in HPV16-positive cervical cancer cell lines (CasKi and $\mathrm{SiHa}$ ) and human cervical carcinoma.

In the present study, we demonstrated that in vivo Klotho expression in cervical carcinoma tissues was decreased as invasiveness became acute compare to its normal counterparts. In vitro studies revealed that Klotho restoration causes a dramatic downregulation of the $\mathrm{Wnt} / \beta$-catenin target gene expression and demonstrate that Klotho significantly inhibit tumor proliferation and invasion by reversal of EMT markers. Based on this, our study indicated that Klotho shows clinical importance in Wnt/ $\beta$-catenin pathway activating HPV16infected cervical cancer.

\section{Materials and methods}

Cell culture. The human cervical cancer cell line $\mathrm{SiHa}$, was purchased from the American Type Culture Collection (Manassas, VA) and was grown in Dulbecco's modified Eagle's medium (WelGene, Seoul) supplemented with $10 \%$ fetal bovine serum, $100 \mu \mathrm{g} / \mathrm{ml}$ penicillin and $100 \mathrm{U} / \mathrm{ml}$ streptomycin. Culture was grown at $37^{\circ} \mathrm{C}$ in a $5 \% \mathrm{CO}_{2}$ atmosphere.

Patient specimens. All uterine cervical tissues were obtained from patients under protocols approved by the institutional review board of Soonchunhyang University (Cheonan, Korea). Tumor tissue $(n=20)$ and adjacent matched normal tissue $(n=20)$ were obtained from women diagnosed with invasive cervical cancer. Histological diagnosis, tumor stage and grade were followed by the Union for International Cancer Control (UICC) classification schemes.

Immunohistochemistry. The uterine cervical tissue was fixed in $10 \%$ neutral buffered formalin for 8-12 h in room temperature and the paraffin blocks was made with standard methods. For immunohistochemical staining of Klotho, the paraffin block sections (4 mm thick) were deparaffinised, rehydrated, placed in $0.01 \mathrm{~mol} / 1$ citrate buffer ( $\mathrm{pH} 6.0$ ), and treated by microwave heating for $15 \mathrm{~min}$. The sections were then preincubated with $0.3 \% \mathrm{H}_{2} \mathrm{O}_{2}$ in methanol for $20 \mathrm{~min}$ at room temperature to quench endogenous peroxidase activity. Subsequently, the sections were immunostained with an UltraTech kit
(Immunotech, Marseille, France) according to the manufacturer's instructions. The sections were pretreated with $1 \%$ bovine serum albumin in phosphate-buffered saline (PBS), and then incubated with anti-Klotho antibody (Sigma, MO, USA) by dilution 1:60 for $1 \mathrm{~h}$ at room temperature. Thereafter, the sections were incubated with biotinylated secondary antibody for $15 \mathrm{~min}$, washed with PBS, and treated with peroxidaseconjugated streptavidin for $20 \mathrm{~min}$. Finally the sections were incubated in 3,3-diaminobenzidine tetrahydrochloride with $0.05 \% \mathrm{H}_{2} \mathrm{O}_{2}$ for $5 \mathrm{~min}$ and then counterstained with haematoxylin. Sections of kidney formalin-fixed paraffin-embedded tissue that had been confirmed to overexpress this protein was used as positive control. The PBS was applied instead of the primary antibody to negative controls.

Reverse transcription-PCR. Total RNA was isolated from vector- or Klotho-transfected SiHa using the Qiagen RNeasy kit (Qiagen) and then reverse transcribed with ImProm-II ${ }^{\mathrm{TM}}$ Reverse Transcription System (Promega), according to the manufacturer's instructions. The cDNA was amplified by PCR using the AccuPower PCR premix (Bioneer, South Korea) with the following primers: Klotho-specific primers were: forward, 5'-ACTCCCCCAGTCAGGTGGCGGTA-3' and reverse, 5'-TGGGCCCGGGAAACCATTGCTGTC-3'. C-myc primers: forward, 5'-ACCAGCAGCGACTCTGAGGAGG AAC-4' and reverse, 5'-TGACCCTCTTGGCAGCAGGATAG TCC-3'; cyclin D1 primers: forward, 5'-ACCTTCGTTGCCC TCTGTGCCACAGATG-3' and reverse, 5'-AGGCCCGGAGG CAGTCCGGGT-3'; E-cadherin primers: forward, 5'-TCCCA TCAGCTGCCCAGAAA-3' and reverse, 5'-TGACTCCTGT GTTCCTGTGTA-3'; N-cadnerin primers: forward, 5'-CAC TGCTCAGGACCCAGAT-3' and reverse, 5'-TAAGCCGAG TGATGGTCC-3'; MMP7 primers: forward, 5'-CGGATGGT AGCAGTCTAGGGATTAAC-3' and reverse, 5'-GGAGTG GAGGAACAGTGCTTATCAATTC-3'; MMP9 primers: forward, 5'-CTTCTCTGGGCGCCAGGT-3' and reverse, 5'-AGGCTTTCTCTCGGTACTGGAAGAC-3'. Human ACTB forward, 5'-CTCGGTGAGGATCTTCATGAGGT AGT-3' and reverse, 5'-CCATCGAGCACGGCATCGTCA CCA-3' was amplified as an endogenous control.

Modification of Klotho expression. The secreted form of human Klotho (sKL) cDNA cloned into pcDNA3.1/V5-His expression vector (Invitrogen) was a generous gift from Michael J. Econs (Indiana University School of Medicine, Indianapolis, IN, USA). SiHa cells were plated at $1 \times 10^{6}$ cells $/ 60-\mathrm{mm}^{3}$ dishes $24 \mathrm{~h}$ prior to transfection and were transfected with $3 \mu \mathrm{g}$ of either sKL expression vector or an empty vector control for $5 \mathrm{~h}$ in serum-free medium using Lipofectamine 2000 (Invitrogen) following the manufacturer's instructions. After replacing the DNA-Lipofectamine complex-containing medium with complete growth medium, transfected cells were incubated for $72 \mathrm{~h}$. After modification of sKL, the altered expression of sKL and $\mathrm{Wnt} / \beta$-catenin signal-related genes was also examined by RT-PCR and immunoblotting.

Immunoblotting. Cells transfected or non-transfected with sKL plasmid were harvested and extracts formed by the addition of lysis buffer. After boiling with $2 \mathrm{X}$ sample buffer, proteins were resolved on $10 \%$ SDS-polyacrylamide gels and 

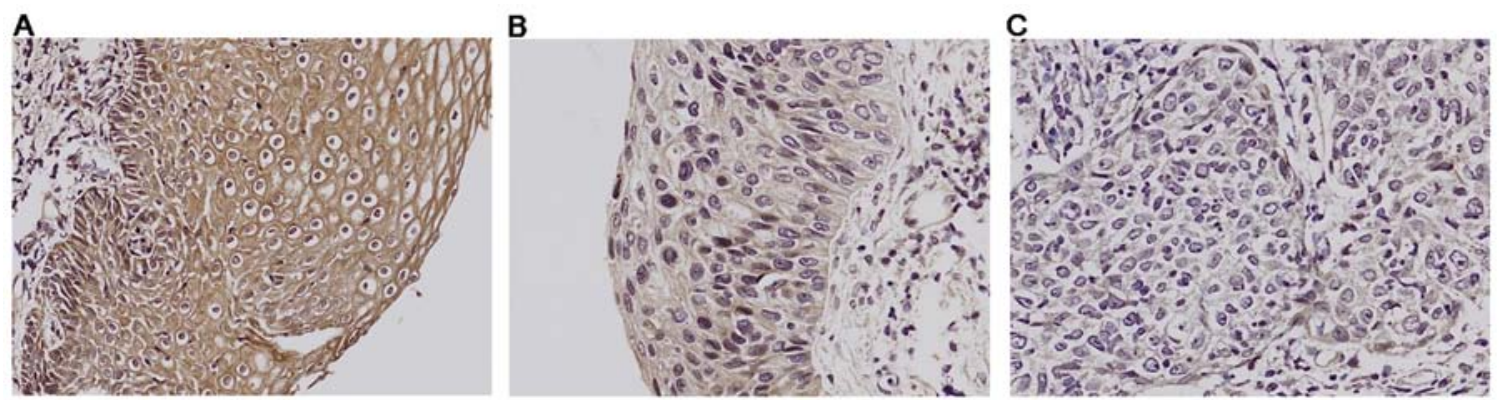

Figure 1. Alteration of Klotho expression in human cervical carcinoma. Immunohistochemical staining of Klotho in human cervical carcinoma with different stages. (A) Strong expression of Klotho in uterine cervical normal stratified squamous epithelium. (B) Mild expression of Klotho in cervical intraepithelial neoplasia stage III. (C) Loss of Klotho expression in invasive squamous cervical carcinoma.

electrotransferred to PVDF membranes (Millipore). Modified sKL expression level and influenced proteins was examined using the antibodies: Polyclonal anti-sKL (T-19, Santa Cruz Biotechnology), cyclin D1 (M-20, Santa Cruz Biotechnology), total $\beta$-catenin (6B3, Cell Signaling), active $\beta$-catenin (no. 9582, Cell Signaling), GSK3 $\beta$ (no. 2199, Epitomics), phospho-GSK3 $\beta$ (no. 2435, Epitomics), E-cadherin (610181, BD Biosciences), N-cadherin (610920, BD), MMP7 (J-22, Santa Cruz Biotechnology), and MMP9 (no. 3852, Cell Signaling). As a loading control, blots were probed with $\gamma$-tubulin (sc7396, Santa Cruz Biotechnology). Immunoreactivity of each protein was visualized using chemiluminescence and recorded on X-ray film.

Wound healing migration. Alteration of cell migration induced by overexpressed sKL was estimated by means of woundhealing migration to measure alteration of two-dimensional cellular movement. pcDNA3.1/HA vector and sKL-transfected SiHa cells were cultured to confluence in $60-\mathrm{mm}^{3}$ dishes. A scratch was made on the monolayer using a sterile pipette tip. At the initiation of the experiment, a microscopic image of the scratch wound was taken at x50 magnification. At $36 \mathrm{~h}$, the same region was imaged. The width of the scratch wounds was measured in Photoshop 7.0. The relative fold change of the scratch wound width at $36 \mathrm{~h}$ after introduction of the scratch compared to the control which control the fold change was calculated as the average of 6 fields.

Matrigel invasion assay and extracellular matrix transition. To examine invasiveness, $2.5 \times 10^{4}$ of vector- or sKL-transfected $\mathrm{SiHa}$ cells per well in serum-free DMEM were placed in the upper chamber. DMEM plus $10 \%$ FBS was placed in the lower chamber as a chemoattractant. Cells were allowed to migrate through a uncoated membrane for $24 \mathrm{~h}$ at $37^{\circ} \mathrm{C}$. Chemoattractant was removed and $4 \mu \mathrm{M}$ Calcein AM Fluorescent Dye (BD Biosciences) was mixed to media or PBS, $0.5 \mathrm{ml}$ dye was added to bottom chamber of 24-well for $1 \mathrm{~h}$ at $37^{\circ} \mathrm{C}$. The amount of migrating cells was determined by measured fluorescence at 494/517 nm (Abs/Em).

\section{Results}

Expression of Klotho in human cervical carcinoma. It has been reported that the decreased expression of Klotho was related to carcinogenesis in breast cancer cells (20), but not in cervical cancer. Our previous study firstly reported that Klotho expression was downregulated in cervical cancer by epigenetic silencing on promoter region (19). According to our previous epigenetic study, Klotho mRNA in human cervical cancer cell lines did not or were slightly expressed, especially in those having higher metastatic potential (CasKi and $\mathrm{SiHa}$ ). In addition, the Klotho mRNA level in human cervical cancer tissues was also downregulated compare to its normal tissues. From this study, we hypothesized that the level of Klotho expression may be in inverse proportion to invasiveness of cervical cancer.

To examine the Klotho expression level and cervical cancer invasiveness, we first examined Klotho expression in a human cervical cancer with different grades by immunohistochemistry. In the uterine cervical normal stratified squamous epithelium, strong Klotho expression was observed in the cytoplasm (Fig. 1A). All normal cervical samples were obtained from women undergoing surgery expressing high Klotho levels. Klotho expression in cervical intraepithelial neoplasia III (CIN III), which is cancer but has not yet invaded deeper tissues, was decreased about half compare to normal counterparts (Fig. 1B). In contrast, there was no Klotho expression detected in invasive cervical cancer tissues (Fig. 1C). Our immunohistochemistry results clearly demonstrate a significant decrease in Klotho protein expression and correlated well with our previous epigenetic silencing of Klotho mRNA as shown by RT-PCR in higher grade of cervical cancer when compared with the normal tissue. It suggests that Klotho downregulation is closely related in cervical invasiveness.

Reversal of epithelial-to-mesenchymal transition by Klotho expression in SiHa cervical cancer cells. The immunohistochemistry data indicated that downregulated Klotho expression might be one of the important factors to increase cervical cancer invasiveness. In addition, our previous study (19) showed that ectopic expression of Klotho with CasKi cells were more compact and adherent to adjacent cells than vector-transfected ones, indicating that Klotho can influence the EMT of cervical cancer cells. Tumor invasion into surrounding tissues requires epithelial cells to lose their polarity and intercellular adhesion (21). E-cadherin is the prime mediator of intercellular adhesion (22) and its 

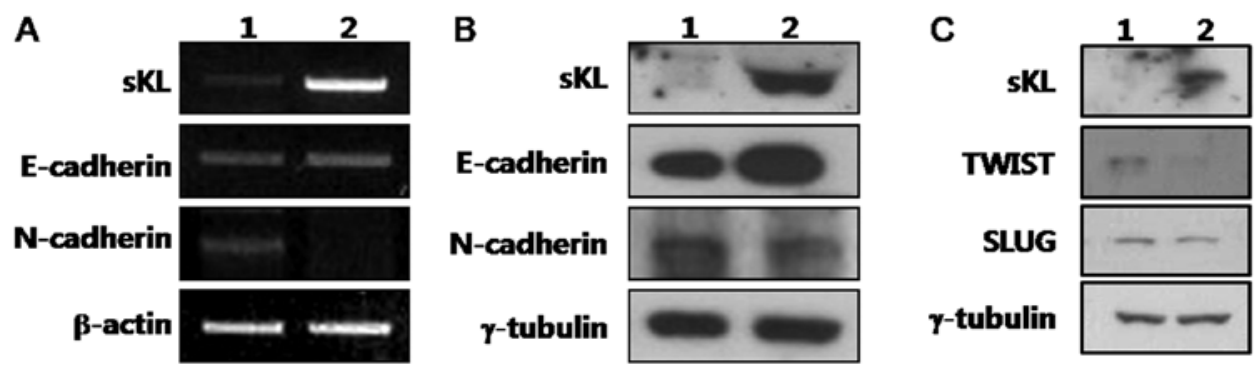

Figure 2. Alteration of epithelial-to-mesenchymal transition (EMT) marker by ectopic expression of Klotho in SiHa cells. (A) Modulation of mRNA expression for epithelial marker and mesenchymal marker by Klotho. (B) Protein expression level of epithelial marker and mesenchymal marker by Klotho restoration. (C) Western blot analysis of transcription factors, Slug and Twist. Lane 1 represents vector-transfected SiHa and lane 2 is for Klotho-transfection. $\beta$-actin and $\gamma$-tubulin were used as a loading control for RT-PCR and western analysis, respectively.

A
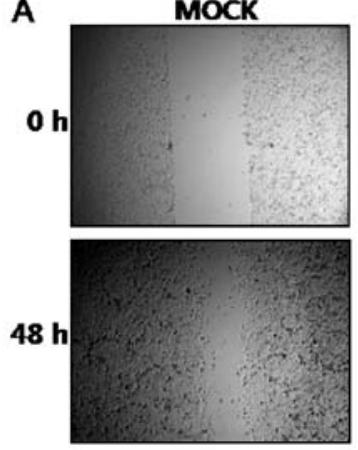

C

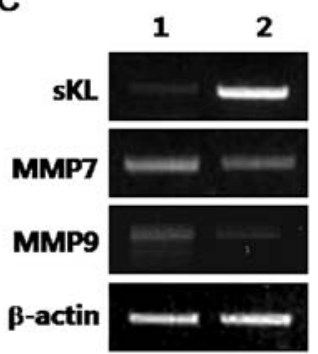

SKL
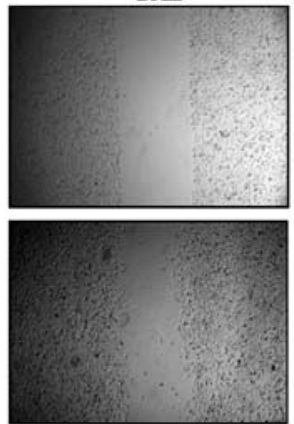

B

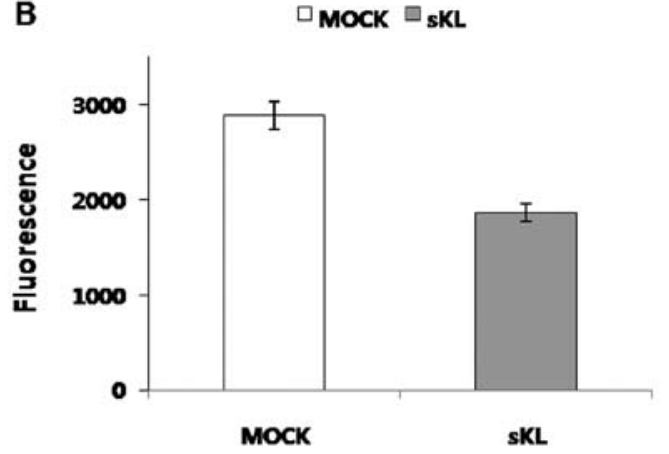

Figure 3. Reduction of cell migratory and invasive capacity and decrease in matrix metalloproteinases by re-expressed Klotho. (A) Representative photomicrographs of wound healing in SiHa cells after transfection of Klotho. (B) Decreased invasive capacity of Klotho-transfected SiHa cells which measured by fluorescence of migrating cells. (C) Modification of matrix metalloproteinase (MMP7 and -9) by ectopic expressed Klotho. RT-PCR analysis was performed for MMP7 and -9. MMP9 expression level was examined by western blot analysis after overexpression of Klotho in SiHa. Lane 1 represents vector-transfected $\mathrm{SiHa}$ and lane 2 is for Klotho-transfection. $\beta$-actin and $\gamma$-tubulin were used as a loading control for RT-PCR and western analysis, respectively.

downregulation is a hallmark of tumor invasion (23). Fig. 2B shows that ectopic expression of Klotho in SiHa cells results in a dramatic increase in the protein levels of E-cadherin and a decrease in $\mathrm{N}$-cadherin. This result suggests that Klotho expression causes a reversal of EMT in cervical cancer cells.

Association with downregulating Slug/Twist expression in EMT modulation by Klotho. Compared to vector control, RT-PCR analysis also shows that Klotho expression in $\mathrm{SiHa}$ cells causes upregulation of E-cadherin, and leads to downregulation of $\mathrm{N}$-cadherin (Fig. 2A). It suggests that the effect of Klotho on the alteration of EMT in SiHa cells may be associated with transcriptional regulation.

Most representative transcription factors involving in EMT regulation are Slug, Snail and Twist which are repressors for E-cadherin gene transcription (24). Furthermore,
Wnt $/ \beta$-catenin signaling has been reported to cause upregulation of the expression of Slug and Twist $(25,26)$ and Klotho can influence its expression acting as Wnt antagonist (19). Fig. 2C demonstrates that the protein levels of Slug and Twist are decreased by Klotho expression. It means that the Klotho-induced reversal of EMT in SiHa cells is associated with downregulation of transcriptional factor Slug/Twist and resultant upregulation of E-cadherin.

Suppression of cellular motility, invasive capacity via downregulation of MMP7 and -9 by Klotho expression. To examine the effect of Klotho on EMT reversal, we studied the effect of Klotho expression on migration of $\mathrm{SiHa}$ cells using a wound healing assay. Fig. 3A shows that Klotho-transfected SiHa cells exhibited slower migration into the wounded area comparing with vector-transfected ones. 


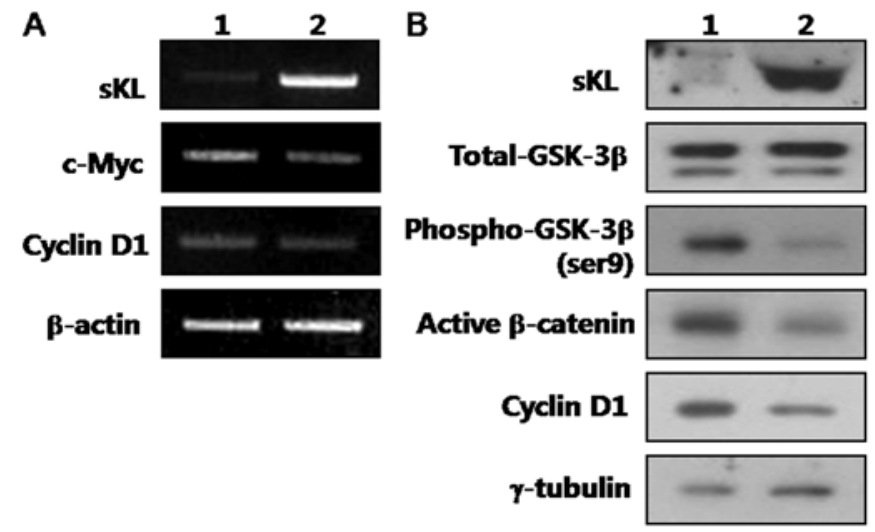

Figure 4. Suppression of Wnt/ $\beta$-catenin pathway by ectopic expression of Klotho. (A) RT-PCR analysis for direct Wnt-target genes such as c-Myc and cyclin D1. (B) Western blot analysis for alteration of GSK-3 $\beta$, phosphorGSK-3 $\beta, \beta$-catenin expression level by overexpressed Klotho. Lane 1 represents vector-transfected $\mathrm{SiHa}$ and lane 2 is for Klotho-transfection. $\beta$-actin and $\gamma$-tubulin were used as a loading control for RT-PCR and western analysis, respectively.

We also examined the in vitro invasiveness of $\mathrm{SiHa}$ cells overexpressing Klotho, or vector transfected cells in a Matrigel invasion assay. Cell motility was measured by average fluorescence of cells migrating through a control, uncoated insert. Klotho-transfected cells exhibited a significant decrease in invasive capacity compared with control cells (Fig. 3B). These data suggest that EMT reversal caused by Klotho expression contributes to the invasiveness of cervical cancer cells.

Matrix metalloproteinases (MMPs) play an important role in cell-matrix interaction and tumor invasion. We therefore studied the effect of Klotho on MMPs expression. Fig. 3C shows that ectopic expression of Klotho in SiHa cells resulted in decreased level of MMP7 and -9. In addition, transcripts of MMP7 and -9 also are decreased in Klotho-transfected SiHa cells similar to the modulation of protein expression levels. It has been reported that MMP activities are correlated with protein expression level of each MMP $(27,28)$. Based on this, Klotho-induced downregulation of MMPs suggests that its activity is also decreased. Altogether, ectopic expression of Klotho suppresses the invasiveness of cervical cancer cells via EMT reversal and downregulation of MMP expression.

Inhibition of canonical Wnt/ $\beta$-catenin pathway by Klotho. Our data indicates that loss of Klotho expression can relate to increased invasiveness through alteration of EMT, cell motility, and MMPs activities. It has been reported that Wnt pathway is involved in cell proliferation, invasion and metastasis through regulation of Wnt target gene expression (29). Altered gene and cellular characteristics by Klotho expression during enhancement of cervical cancer was observed mostly in the Wnt pathway. In addition, we previously found Klotho is Wnt antagonist, so we analyzed the expression of Klotho, GSK3 $\beta$, $\beta$-catenin and specific Wnt $\beta$-catenin direct transcriptional target genes with representative roles in tumor progression and invasion. Fig. 4 shows that ectopic expression of Klotho in SiHa cells caused a decreased expression of phospho-GSK3 $\beta$ at serine-9 without changing the levels of its total protein. In addition, alteration of GSK $3 \beta$ reduced the active form of $\beta$-catenin (ABC), which is dephosphorylated on S37 or T41 residues.

c-Myc seems to be essential for sustaining proliferation of human tumor cells (30). Importantly, Klotho overexpression resulted in a marked downregulation of c-Myc mRNA being a direct transcriptional Wnt/ $\beta$-catenin target (Fig. 4A). The expression of cyclin D1, another direct transcriptional Wnt/ $\beta$-catenin target with major roles in cell proliferation $(31,32)$ was also markedly downregulated in Klotho-transfected SiHa cells (Fig. 4). Together, these results indicate that Klotho expression inhibits human cervical cancer invasion by having a major regulatory role on the expression of specific $\beta$-catenin direct transcriptional targets.

\section{Discussion}

It has been reported that the first etiology of cervical cancer is HPV infection and it potentially activates Wnt/ $\beta$-catenin signaling pathway to promote tumorigenesis. Klotho is known as a Wnt antagonist and it is downregulated mainly by promoter hypermethylation in several human tumors, including lung, breast, pancreatic, colon and cervical cancer (19,33-35). Although it has been reported that Klotho acts as tumor suppressor and inhibits cancer cell proliferation in many cancers (36), little is known about its potential effect on metastatic cervical tumor and the process of this tumor metastasis. We reported here that Klotho expression resulted in a decreased capacity of cell migration and invasion. This was confirmed by immunohistochemistry data using human cervical cancer tissues. Normal cervical tissues have very strong Klotho expression and it was decreased to about half in CIN III grade. In contrast, no or very low level of Klotho was observed in invasive cervical cancer tissues. This suggests that the loss of Klotho in cervical cancer may contribute to its metastatic potential. Based on our study, the action mechanism of Klotho is associated with a reversal of the EMT process via downregulation of E-cadherin expression, upregulation of N-cadherin expression and decreased activity of MMP7 and -9.

EMT is characterized by increased migratory features, decreased epithelial cell adhesion, loss of cytoskeleton components and acquisition of mesenchymal components (37). A hallmark of EMT is the loss of E-cadherin expression (37). Our study showed that overexpression of Klotho in SiHa cells changed EMT by both increase of E-cadherin and decrease of $\mathrm{N}$-cadherin. The increase of E-cadherin with ectopic expression of Klotho was associated with downregulation of the transcriptional repressors Slug and Twist. In a recent study Doi et al (38) demonstrated that in renal fibrosis and metastatic cells restoring the expression of Klotho leads to inhibition of TGF- $\beta 1$-induced EMT. It means that $\mathrm{Wnt} / \beta$-catenin pathway may also participate in regulation of the EMT process in cancer progression and that Klotho may interfere with it.

Downregulation of E-cadherin leads to the loss of intercellular adhesion (23). SiHa cells transfected with Klotho showed a marked decrease in the expression of active $\beta$-catenin, c-Myc and cyclin D1. These data suggest that Klotho blocks Wnt/ $\beta$-catenin pathway and consequently inhibits cervical cancer cell proliferation. In addition, it can alter the invasive behavior of cervical cancer cells. According to our wound healing assay, 
Klotho-transfected SiHa cells which have increased E-cadherin moved slower into wound lesions. E-cadherin binds directly to $\beta$-catenin (22) and this binding is prerequisite for formation of cell-cell adhesion, which prevents tumor invasion. Concurring with this, E-cadherin expression correlates negatively with progression of cervical intraepithelial neoplasia (39). Overall, it strongly support the hypothesis that Klotho blocks human cervical cancer progression, and its re-expression has the possibility to change patient's prognosis. Taken together, Klotho is a potent invasion suppressor as strong as tumor suppressor. Based on our results, the effect of Klotho can be a result of synergistic convergence of multiple regulatory pathways, including induction of apoptosis, decrease in proliferation, and angiogenesis, due to the $\mathrm{Wnt} / \beta$-catenin signaling pathway being immensely influential. Therefore, our further studies in progress will determine which Klotho could cause concomitant suppression of tumor progression. However, it still remains unknown that the inhibitory mechanism of Klotho in Wnt/ $\beta$-catenin signaling especially in cervical cancer. It has been reported that Klotho can interact with Wnt ligands such as Wnt5a, and Wnt3 to suppress metastasis in melanoma or aging $(36,40)$. Therefore, our future study will be focused on the processes of $\mathrm{Wnt} / \beta$-catenin pathway by Klotho.

In conclusion, we report for the first time that loss of Klotho leads to aberrant activation of $\mathrm{Wnt} / \beta$-catening signaling in human cervical cancer. It was also shown that re-expression of Klotho causes a remarkable inhibition of the Wnt/ $\beta$-catenin pathway blocking tumor invasion. Thus, our findings revealed more information on the unknown and novel invasion-suppressive signaling mechanisms of Klotho, and also emphasize the potential therapeutic value of Klotho in human cervical cancer treatment.

\section{Acknowledgements}

This study was supported by a grant funded by Sookmyung Women's University (2011).

\section{References}

1. Parkin DM and Bray F: The burden of HPV-related cancers. Vaccine 24 (Suppl 3): Chapter 2: S3/11-25, 2006.

2. Woodman CB, Collins SI and Young LS: The natural history of cervical HPV infection: unresolved issues. Nat Rev Cancer 7 : 11-22, 2007.

3. Parkin DM, Bray F, Ferlay J and Pisani P: Estimating the world cancer burden: Globocan 2000. Int J Cancer 94 153-156, 2001.

4. Uren A, Fallen S, Yuan H, Usubutun A, Kucukali T, Schlegel R, et al: Activation of the canonical Wnt pathway during genital keratinocyte transformation: a model for cervical cancer progression. Cancer Res 65: 6199-6206, 2005.

5. Perez-Plasencia C, Duenas-Gonzalez A and Alatorre-Tavera B: Second hit in cervical carcinogenesis process: involvement of wnt/beta catenin pathway. Int Arch Med 1: 10, 2008.

6. MacDonald BT, Tamai K and $\mathrm{He} \mathrm{X}$ : Wnt $/ \beta$-catenin signaling: components, mechanisms, and diseases. Dev Cell 17: 9-26, 2009.

7. Paul S and Dey A: Wnt signaling and cancer development: therapeutic implication. Neoplasma 55: 165-176, 2008.

8. Shinohara A, Yokoyama Y, Wan X, Takahashi Y, Mori Y, Takami T, et al: Cytoplasmic/nuclear expression without mutation of exon 3 of the $\beta$-catenin gene is frequent in the development of the neoplasm of the uterine cervix. Gynecol Oncol 82: 450-455, 2001.
9. Ueda M, Gemmill RM, West J, Winn R, Sugita M, Tanaka N, et al: Mutations of the $\beta$ - and $\gamma$-catenin genes are uncommon in human lung, breast, kidney, cervical and ovarian carcinomas. $\mathrm{Br}$ J Cancer 85: 64-68, 2001.

10. Chung MT, Sytwu HK, Yan MD, Shih YL, Chang CC, Yu MH, et al: Promoter methylation of SFRPs gene family in cervical cancer. Gynecol Oncol 112: 301-306, 2009.

11. Lee EJ, Jo M, Rho SB, Park K, Yoo YN, Park J, et al: Dkk3, downregulated in cervical cancer, functions as a negative regulator of $\beta$-catenin. Int J Cancer 124: 287-297, 2009.

12. Kuro-o M, Matsumura Y, Aizawa H, Kawaguchi H, Suga T, Utsugi T, et al: Mutation of the mouse klotho gene leads to a syndrome resembling ageing. Nature 390: 45-51, 1997.

13. Imura A, Iwano A, Tohyama O, Tsuji Y, Nozaki K, Hashimoto N, et al: Secreted Klotho protein in sera and CSF: implication for post-translational cleavage in release of Klotho protein from cell membrane. FEBS Lett 565: 143-147, 2004.

14. Chen CD, Podvin S, Gillespie E, Leeman SE and Abraham CR: Insulin stimulates the cleavage and release of the extracellular domain of Klotho by ADAM10 and ADAM17. Proc Natl Acad Sci USA 104: 19796-19801, 2007.

15. Mian IS: Sequence, structural, functional, and phylogenetic analyses of three glycosidase families. Blood Cells Mol Dis 24: 83-100, 1998.

16. Kurosu H, Ogawa Y, Miyoshi M, Yamamoto M, Nandi A, Rosenblatt KP, et al: Regulation of fibroblast growth factor-23 signaling by klotho. J Biol Chem 281: 6120-6123, 2006.

17. Tao Y, Pinzi V, Bourhis J and Deutsch E: Mechanisms of disease: signaling of the insulin-like growth factor 1 receptor pathway therapeutic perspectives in cancer. Nat Clin Pract Oncol 4: 591-602, 2007.

18. Mattarocci S, Abbruzzese C, Mileo AM, Visca P, Antoniani B, Alessandrini G, et al: Intracellular presence of insulin and its phosphorylated receptor in non-small cell lung cancer. J Cell Physiol 221: 766-770, 2009.

19. Lee J, Jeong DJ, Kim J, Lee S, Park JH, Chang B, et al: The antiaging gene $K L O T H O$ is a novel target for epigenetic silencing in human cervical carcinoma. Mol Cancer 9: 109, 2010.

20. Wolf I, Levanon-Cohen S, Bose S, Ligumsky H, Sredni B, Kanety $\mathrm{H}$, et al: Klotho: a tumor suppressor and a modulator of the IGF-1 and FGF pathways in human breast cancer. Oncogene 27: 7094-7105, 2008.

21. Polyak K and Weinberg RA: Transitions between epithelial and mesenchymal states: acquisition of malignant and stem cell traits. Nat Rev Cancer 9: 265-273, 2009.

22. Huber AH and Weis WI: The structure of the $\beta$-catenin/E-cadherin complex and the molecular basis of diverse ligand recognition by $\beta$-catenin. Cell 105: 391-402, 2001.

23. Laux H, Tomer R, Mader MT, Smida J, Budczies J, Kappler R, et al: Tumor-associated E-cadherin mutations do not induce Wnt target gene expression, but affect E-cadherin repressors. Lab Invest 84: 1372-1386, 2004.

24. Yang J and Weinberg RA: Epithelial-mesenchymal transition: at the crossroads of development and tumor metastasis. Dev Cell 14: 818-829, 2008.

25. Vallin J, Thuret R, Giacomello E, Faraldo MM, Thiery JP and Broders F: Cloning and characterization of three Xenopus slug promoters reveal direct regulation by Lef/ $\beta$-catenin signaling. J Biol Chem 276: 30350-30358, 2001.

26. Howe LR, Watanabe O, Leonard J and Brown AM: Twist is up-regulated in response to Wnt 1 and inhibits mouse mammary cell differentiation. Cancer Res 63: 1906-1913, 2003.

27. Marchenko GN, Marchenko ND, Leng J and Strongin AY: Promoter characterization of the novel human matrix metalloproteinase-26 gene: regulation by the T-cell factor-4 implies specific expression of the gene in cancer cells of epithelial origin. Biochem J 363: 253-262, 2002.

28. Wu B, Crampton SP and Hughes CC: Wnt signaling induces matrix metalloproteinase expression and regulates $\mathrm{T}$ cell transmigration. Immunity 26: 227-239, 2007.

29. Yee DS, Tang Y, Li X, Liu Z, Guo Y, Ghaffar S, et al: The Wnt inhibitory factor 1 restoration in prostate cancer cells was associated with reduced tumor growth, decreased capacity of cell migration and invasion and a reversal of epithelial to mesenchymal transition. Mol Cancer 9: 162, 2010.

30. Wang H, Mannava S, Grachtchouk V, Zhuang D, Soengas MS, Gudkov AV, et al: c-Myc depletion inhibits proliferation of human tumor cells at various stages of the cell cycle. Oncogene 27: 1905-1915, 2008. 
31. Fu M, Wang C, Li Z, Sakamaki T and Pestell RG: Minireview: Cyclin D1: normal and abnormal functions. Endocrinology 145: 5439-5447, 2004.

32. Kainz C, Kohlberger P, Tempfer C, Sliutz G, Gitsch G, Reinthaller A, et al: Prognostic value of CD44 splice variants in human stage III cervical cancer. Eur J Cancer 31A: 1706-1709, 1995.

33. Wang L, Wang X, Jie P, Lu H, Zhang S, Lin X, et al: Klotho is silenced through promoter hypermethylation in gastric cancer. Am J Cancer Res 1: 111-119, 2011.

34. King GD, Rosene DL and Abraham CR: Promoter methylation and age-related downregulation of Klotho in rhesus monkey. Age (Dordr): Sep 16, 2011 (Epub ahead of print).

35. Pan J, Zhong J, Gan LH, Chen SJ, Jin HC, Wang X, et al: Klotho, an anti-senescence related gene, is frequently inactivated through promoter hypermethylation in colorectal cancer. Tumour Biol 32: 729-735, 2011

36. Liu H, Fergusson MM, Castilho RM, Liu J, Cao L, Chen J, et al: Augmented Wnt signaling in a mammalian model of accelerated aging. Science 317: 803-806, 2007.
37. Kang Y and Massague J: Epithelial-mesenchymal transitions: twist in development and metastasis. Cell 118: 277-279, 2004

38. Doi S, Zou Y, Togao O, Pastor JV, John GB, Wang L, et al: Klotho inhibits transforming growth factor-beta1 (TGF-beta1) signaling and suppresses renal fibrosis and cancer metastasis in mice. J Biol Chem 286: 8655-8665, 2011.

39. Branca M, Giorgi C, Ciotti M, Santini D, Di Bonito L, Costa S, et al: Down-regulation of E-cadherin is closely associated with progression of cervical intraepithelial neoplasia (CIN), but not with high-risk human papillomavirus (HPV) or disease outcome in cervical cancer. Eur J Gynaecol Oncol 27: 215-223, 2006.

40. Camilli TC, Xu M, O'Connell MP, Chien B, Frank BP, Subaran $\mathrm{S}$, et al: Loss of Klotho during melanoma progression leads to increased filamin cleavage, increased Wnt5A expression, and enhanced melanoma cell motility. Pigment Cell Melanoma Res 24: 175-186, 2011 